\title{
Xom interacts with and stimulates transcriptional activity of LEF1/TCFs: implications for ventral cell fate determination during vertebrate embryogenesis
}

\author{
Hong $\mathrm{Gao}^{1,2}$, Bin $\mathrm{Wu}^{1}$, Roger Giese ${ }^{2}$, Zhenglun $\mathrm{Zhu}^{1}$ \\ ${ }^{1}$ Department of Medicine, Brigham and Women's Hospital, Harvard Medical School, Boston, MA 02115, USA; ${ }^{2}$ Department of Phar- \\ maceutical Sciences, Northeastern University, Boston, MA, USA
}

LEF1/TCFs are high mobility group box-containing transcriptional factors mediating canonical Wnt/ $\beta$-catenin signaling during early embryogenesis and tumorigenesis. $\beta$-Catenin forms a complex with LEF1/TCFs and transactivates LEF1/TCF-mediated transcriptions during dorsalization. Although LEF-mediated transcription is also implicated in ventralization, the underlying molecular mechanism is not well understood. Using the vertebrate Xenopus laevis model system, we found that Xom, which is a ventralizing homeobox protein with dual roles of transcriptional activation and repression, forms a complex with LEF1/TCF through its homeodomain and transactivates LEF1/TCF-mediated transcription through its N-terminal transactivation domain (TAD). Our data show that Xom lacking the N-terminal TAD fails to transactivate ventral genes, such as BMP4 and Xom itself, but retains the ability to suppress transcriptional activation of dorsal gene promoters, such as the Goosecoid promoter, indicating that transactivation and repression are separable functions of Xom. It has been postulated that Xom forms a positive re-enforcement loop with BMP4 to promote ventralization and to suppress dorsal gene expression. Consistent with an essential role of Xom transactivation of LEF1/TCFs during early embryogenesis, we found that expression of the dominant-negative Xom mutant that lacks the TAD fails to re-enforce the ventral signaling of BMP4 and causes a catastrophic effect during gastrulation. Our data suggest that the functional interaction of Xom and LEF1/TCF-factors is essential for ventral cell fate determination and that LEF1/TCF factors may function as a point of convergence to mediate the combined signaling of $\mathrm{Wnt} / \beta$-catenin and BMP4/Xom pathways during early embryogenesis.

Keywords: Xom, Vent, Wnt, BMP4, $\beta$-catenin, LEF1/TCFs, dorsoventral patterning

Cell Research (2007) 17:345-356. doi: 10.1038/cr.2007.20; published online 3 April 2007

\section{Introduction}

Xom (also called Vent2, Vox and Xbr-1), a cell fate determination factor of the Vent family of homeobox genes, is both a transcriptional repressor and an activator [1-4]. During early embryogenesis, Xom is implicated in the formation of ventral mesoderm and in defining the dorsoventral patterning [5, 6]. Zygotic Xom transcription starts

Correspondence: Zhenglun Zhu

Tel: +1-617-732-5467; Fax: +1-617-730-5807

E-mail: zzhu@partners.org

Received 16 February 2007; revised 5 March 2007; accepted 6 March 2007; published online 3 April 2007 after midblastula transition (MBT) and distributes from a more ubiquitous expression pattern during the early gastrula stage to the ventral-lateral regions as gastrulation proceeds $[1,3]$. The expression of Xom appears to be positively regulated by signals from the ventral signal center, such as BMP4, but negatively regulated by dorsal-specific genes, such as Goosecoid (Gsc) and noggin $[1,2]$. Xom expression in turn contributes to the formation of the dorsoventral pattern by promoting the expression of ventral genes such as BMP4 and the Vent genes, and by inhibiting the expression of dorsal-organizer genes such as Gsc and chordin [2, $3,7]$. To exert its transcriptional repressor function, Xom binds directly to the distal element in promoters of dorsal specific genes (such as the Gsc promoter), and inhibits their 
transcription [8]. The molecular mechanism underlying the transcriptional activation function of Xom, however, remains to be fully defined.

The LEF1/TCFs are a family of high mobility group (HMG) transcriptional factors that possess no intrinsic transcriptional activities. Rather, the LEF1/TCF-mediated transcription activities are tightly controlled by their associated factors [9]. In a non-induction state, the LEF1/ TCFs are associated with transcriptional repressors, such as Grouch and CtBP, which maintain the LEF1/TCF-mediated transcription in a repressed state [10-14]. During early embryogenesis, local enrichment of $\beta$-catenin in the future dorsal side of embryos allows it to interact with LEF1/TCFs and to induce the expression of dorsal-specific genes, such as Siamois, Twin and Xnr [15-18]. Besides determination of cell fate during early embryogenesis, excessive activation of LEF1/TCF-mediated transcription by $\beta$-catenin has also been implicated as the initial step of malignant transformation in a variety of cancers [19]. Since LEF1/TCFs represent the transcriptional action point of Wnt/ $\beta$-catenin signaling, LEF1/TCF-responsive luciferase reporter activity has generally been utilized as an indicator of $\mathrm{Wnt} / \beta$-catenin activities.

The role of LEF1/TCFs in ventral cell fate determination is less clear, although several studies have implicated their potential involvement. For example, expression profiling showed that members of LEF1/TCF family are broadly distributed in ventral-posterior regions [20, 21]. Mutagenesis studies revealed that LEF1-/-TCF-/- mice carry caudal defects with neural expansion [22] and that loss of function of LEF1 leads to ventral rather than dorsal defects in Xenopus [23]. Consistent with the possible involvement of LEF1/TCFs in ventral cell fate determination, promoter analysis revealed that many ventral genes, such as Xom and Bambi, contain LEF1/TCF binding sites [24]. Mutations of the LEF1/TCF binding sites in these ventral genes cause significant inhibition of their responsiveness to BMP4 signaling [24].

To further define the role of LEF1/TCF factors in ventral cell fate determination, we examined the potential effect of Xom on LEF1/TCF-mediated transcription. We found that Xom forms a complex with LEF1/TCF factors and transactivates LEF1/TCF-mediated transcription. To explore the molecular mechanism of Xom transactivation of LEF1/TCF-mediated transcription, we found that similar to the case of LEF1/TCF transactivation by $\beta$-catenin [25], the domains of Xom involved in LEF1/TCF interaction and transactivation are different. We have located the TAD of Xom to its N-terminal region, which is separated from the transcriptional repressor domain of Xom. We have further defined that the C-terminal region of LEF1 is involved in interaction with Xom. We found that the Xom TAD is required for transactivation of ventral specific genes, such as BMP4 and Xom itself. Expression of a dominant-negative Xom that lacks the TAD inhibits BMP4 signaling and disrupts embryogenesis at the gastrulation stage. Our data suggest that the functional interaction between Xom and LEF1/TCF factors plays an essential role in ventral cell fate determination during early embryogenesis. We therefore propose a model in which the LEF1/TCF transcription factors, previously known to be the transcriptional action point of $\mathrm{Wnt} / \beta$-catenin signaling, may serve as a point of convergence to mediate the combined signaling effect of $\mathrm{BMP} / \mathrm{Xom}$ and $\mathrm{Wnt} / \beta$-catenin pathways during dorsalventral patterning.

\section{Materials and Methods}

\section{Preparation of Xenopus embryos, microinjection and lucif- erase assays}

Protocols for handling embryos and for luciferase assays were essentially as described previously [26]. For a typical cellular luciferase assay, $2 \times 10^{5}$ cells were split into 12 -well cell-culture plates, incubated for $24 \mathrm{~h}$ and then transfected with $3 \mu \mathrm{l}$ of liposome transfection reagent (TransIT1, Mirus), using $1 \mu \mathrm{g}$ of DNA plasmids of selected genes and $0.3 \mu \mathrm{g}$ of reporter plasmids (HeLa cells require one-third while 293T cells require one-sixth of the DNA and liposome amount). At $48 \mathrm{~h}$ post-transfection, cells were washed with PBS, lysed with $1 \times$ cell lysis buffer (Promega cell lysis buffer), scraped and collected. After incubation on ice for $30 \mathrm{~min}$, cells were cleared by centrifugation at $12000 \times g$ for $15 \mathrm{~s}$, and supernatants were transferred to a new tube; then $20 \mu$ l of the cell lysate was mixed with $100 \mu$ l of Luciferase Assay reagent (Promega), and the luciferase activity was measured by a TR717 Microplate luminometer (Applied Biosystem).

\section{Plasmids and recombinant proteins}

Xenopus LEF1, LEF1 $\Delta \mathrm{HMG}$, LEF1 $\Delta \mathrm{N} 60$, LEF1 $\Delta \mathrm{N} 160$, Xom, and Xom deletion mutants were subcloned by the polymerase chain reaction based technique into the $\mathrm{pCS} 2+$ and PGEX4T3 vectors, and the constructs were verified by in vitro translation and sequencing. pGL3-OT and pGL3-OF were generous gifts from Dr B Volgestein; p2.4BMP4-Luc was a generous gift from Dr J Feng; pGL3 promoter MSX2 (WT) and pGL3 promoter MSX2-SDM-600/-766 were generous gifts from Dr C Sirard.

\section{Preparation of nuclear and cytoplasmic extracts}

Cells $\left(4 \times 10^{6}\right)$ were trypsinized and washed twice with PBS and pelleted by centrifugation. Total protein was obtained by lysing the cells in $150 \mu 1$ of RIPA buffer $(150 \mathrm{mM} \mathrm{NaCl}, 1 \% \mathrm{NP} 40,0.5 \% \mathrm{DOC}$, $0.1 \%$ SDS, $50 \mathrm{mM}$ Tris $\mathrm{pH} 8.0$ ) containing $1 \times$ proteinase inhibitors (Roche, proteinase inhibitor cocktail). Cytoplasmic proteins were obtained by incubation of $2 \times 10^{6}$ cells with $150 \mu$ of hypotonic buffer (0.05\% NP40, $10 \mathrm{mM}$ HEPES pH 7.9, $1.5 \mathrm{mM} \mathrm{MgCl}_{2}, 10 \mathrm{mM} \mathrm{KCl}$, $1 \mathrm{mM}$ DTT) containing $1 \times$ proteinase inhibitors on ice for $10 \mathrm{~min}$ followed by centrifugation at 4000 r.p.m. for $2 \mathrm{~min}$. The supernatants were collected and used as cytoplasmic protein fractions. The nuclear pellets were washed twice with PBS. Nuclear proteins were obtained by incubating the pellet on ice for $60 \mathrm{~min}$ in $150 \mu \mathrm{l}$ of RIPA buffer. Levels of $\beta$-catenin in fractioned cellular extracts were determined 
by western blotting using a specific antibody (Takara Bio).

\section{GST pull-down assay}

Five micrograms of GST fusion proteins and $5 \mu 1$ of ${ }^{35} \mathrm{~S}$-labeled proteins derived from in vitro translation were mixed with $20 \mu \mathrm{l}$ of $50 \%$ glutathione Sepharose 4B beads in $500 \mu \mathrm{l}$ of binding buffer (50 mM Tris pH 8.0, $100 \mathrm{mM} \mathrm{NaCl}, 50 \mathrm{mM} \mathrm{KCl}, 5 \mathrm{mM} \mathrm{MgCl}$, $1 \mathrm{mM}$ DTT, $10 \%$ glycerol and $0.2 \% \mathrm{NP} 40$ ). The mixtures were incubated at $4{ }^{\circ} \mathrm{C}$ for $3 \mathrm{~h}$ and washed with $1 \times$ PBS plus $0.2 \% \mathrm{NP} 40$ four times. Bound material was released with $2 \times$ sample buffer, boiled at $95{ }^{\circ} \mathrm{C}$ for $5 \mathrm{~min}$, centrifuged briefly, and revealed by SDS-PAGE and autoradiography.

\section{Co-immunoprecipitation}

The affinity protein $\mathrm{G}$ beads were prepared by mixing $20 \mu \mathrm{l}$ of protein G plus-Agarose beads (Santa Cruz Biotechnology) with 1 $\mu \mathrm{g}$ of corresponding antibody. A total of $2 \times 10^{6}$ of cells were lysed with $1 \times$ cell lysis buffer (Promega) containing $1 \times$ protease inhibitor reagent (Roche), incubated on ice for $30 \mathrm{~min}$, sonicated briefly and centrifuged at $12000 \times g$ for $5 \mathrm{~min}$ at $4{ }^{\circ} \mathrm{C}$. The supernatants were further cleaned with $20 \mu \mathrm{l}$ of untreated protein $\mathrm{G}$ plus-Agarose beads and $2 \mu \mathrm{g}$ of pre-immune serum for $2 \mathrm{~h}$ at $4{ }^{\circ} \mathrm{C}$. The supernatants were then mixed with $20 \mu \mathrm{l}$ of prelabeled protein $\mathrm{G}$ plus-Agarose beads at $4{ }^{\circ} \mathrm{C}$ overnight. The beads were washed four times with PBS plus $0.2 \%$ NP40. Bound proteins were released by $2 \times$ sample buffer, boiled at $95^{\circ} \mathrm{C}$ for $5 \mathrm{~min}$, centrifuged briefly and revealed by western blot analysis with specific antibodies.

\section{Histological staining}

Embryos were fixed with 3.7\% formaldehyde, 0.1 M MOPS
(pH 7.4), 2 mM EGTA, and $1 \mathrm{mM} \mathrm{MgSO}_{2}$ at indicated stages. The embryos were then embedded in paraffin and sectioned at a $10 \mathrm{~mm}$ thickness. The sections were stained with hematoxylin and eosin (H\&E), and subjected to histological analysis. The sections were further stained with $2 \mu \mathrm{g} / \mathrm{ml}$ of 4',6-diamidino-2-phenylindole, dihydrochloride (DAPI) (Invitrogen) to reveal the nuclei of embryonic cells.

\section{Analysis of gene expression by real-time PCR}

Total RNA was extracted by Trisol methods. Eight embryos from each treatment group were pooled and homogenized in $1 \mathrm{ml}$ of Trizol (Invitrogen). Chloroform $(200 \mu \mathrm{l})$ was then added to the sample. After vortex mixing, the samples were centrifuged at 12000 r.p.m. for $1 \mathrm{~min}$ and the supernatants were collected in new tubes. Isoproponol $(500 \mu \mathrm{l})$ was added to each sample, mixed and kept at RT for $10 \mathrm{~min}$. Samples were then centrifuged for $30 \mathrm{~min}$. The pellets were washed twice with $70 \%$ ethanol, air dried and suspended in $100 \mu \mathrm{l}$ of $\mathrm{ddH}_{2} \mathrm{O}$. The final RNA concentration was determined by measurement at $\mathrm{OD}_{260}$. First-strand cDNA was synthesized with the SuperScript first-strand synthesis system (Invitrogen) according to the manufacturer's protocol. Briefly, $2.5 \mu \mathrm{g}$ of total RNA from each sample was used. The final volume of the RT product was $20 \mu \mathrm{l}$ and was diluted to $200 \mu \mathrm{l}(10 \times$ dilution $) ; 8 \mu \mathrm{l}$ of the diluted RT product was used for real-time PCR using LightCycler System (Roche) and LightCycler FastStart DNA Master SYBR Green I, according to the manufacturer's instructions. The relative levels of gene expression were calculated by the formula relative gene expression $=2-\Delta \mathrm{Cd}$ $(\Delta \mathrm{Cd}=$ cycle of the specific gene-cycle of the reference Histone-4 gene). Each sample was carried out in triplicate. The sequences of the primers are available upon request.
A TOP/FOP assay in Xenopus embryos

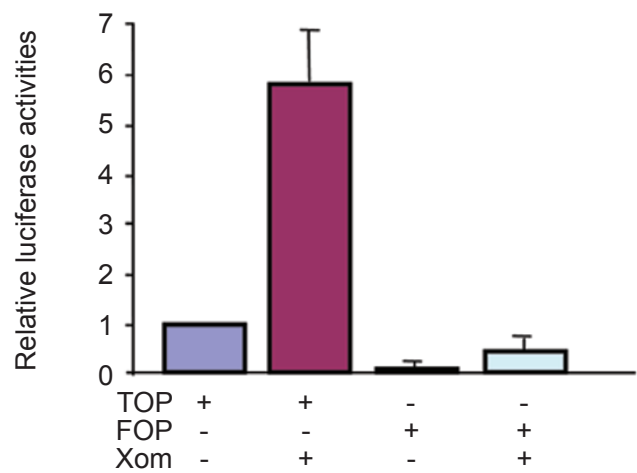

B

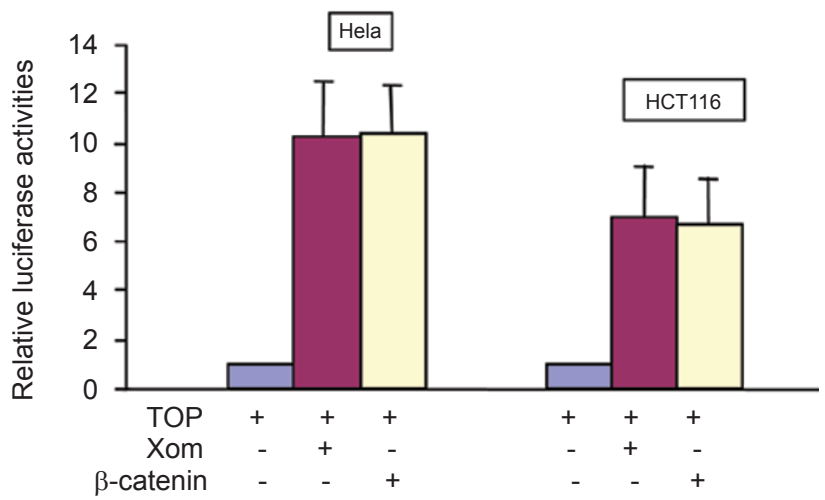

Figure 1 Activation of LEF1/TCF-mediate transcription by Xom. (A) Xom activates the LEF1/TCF reporter (TOPflash) in embryonic cells. TOPflash construct ( $100 \mathrm{pg}$ ) was injected into one of the two blastomeres either alone or in conjunction with 0.4 ng of mRNA encoding Xom. Luciferase activity was determined after stage 10, and the luciferase activity in embryos injected with TOPflash alone was designated as one unit. FOPflash plasmid was used as the negative control. (B) Xom activates LEF1/TCF reporter in HeLa and HCT116 cells. TOPflash construct was transiently transfected into the indicated cells either alone or in conjunction with plasmids encoding Xom and/or $\beta$-catenin as indicated. The luciferase activity was determined $48 \mathrm{~h}$ after transfection. In each cell type, luciferase activities in cells expressing TOPflash alone were defined as one unit. Error bars indicate standard deviations of representative experiments carried out in triplicate. 


\section{Results}

Xom transactivates LEF1/TCF-mediated transcription

Xom is a major ventral cell fate determination factor of the BMP4 signaling pathway. Using the TOPflash assay, a previous investigation has located LEF1/TCF-mediated transcriptional activities in the ventral-posterior side of embryos [27]. By injecting TOPflash plasmid into the two ventral blastomeres at the four-cell stage and examining luciferase activities during gastrulation stage, we have also confirmed the LEF1/TCF-mediated transcriptional activities on the ventral side of embryos during early embryogenesis (data not shown, the pGL3-OT, which contains three copies of the optimal TCF binding motif, was a generous gift from Dr B Vogelstein) [28]. To explore a potential inter-relationship between Xom expression and LEF/TCF-mediated transcription, we examined the effect of Xom expression on LEF1/TCF-mediated transcription using the TOPflash-luciferase assay. When mRNA encoding Xom was co-injected with the TOPflash reporter construct into Xenopus embryos at the two-cell stage, expression of Xom enhanced the TOPflash reporter transcription by sevenfold as compared with embryos injected with a construct of TOPflash alone (Figure 1A). The trans-activating effect of Xom on the TOPflash reporter was specific, since expression of Xom did not activate the control FOPflash reporter (Figure 1A) (pGL3-OF, also a gift from Dr Vogelstein), which contains mutations at the LEF/TCF binding sites [28]. To determine whether Xom transactivation of LEF1/TCF-mediated transcription is dependent on cellular contexts of embryos, we also studied the effect of Xom expression on LEF1/TCF-mediated transcription in non-embryonic cells. When plasmids for Xom and TOPflash were co-transfected into HeLa and HCT116

A
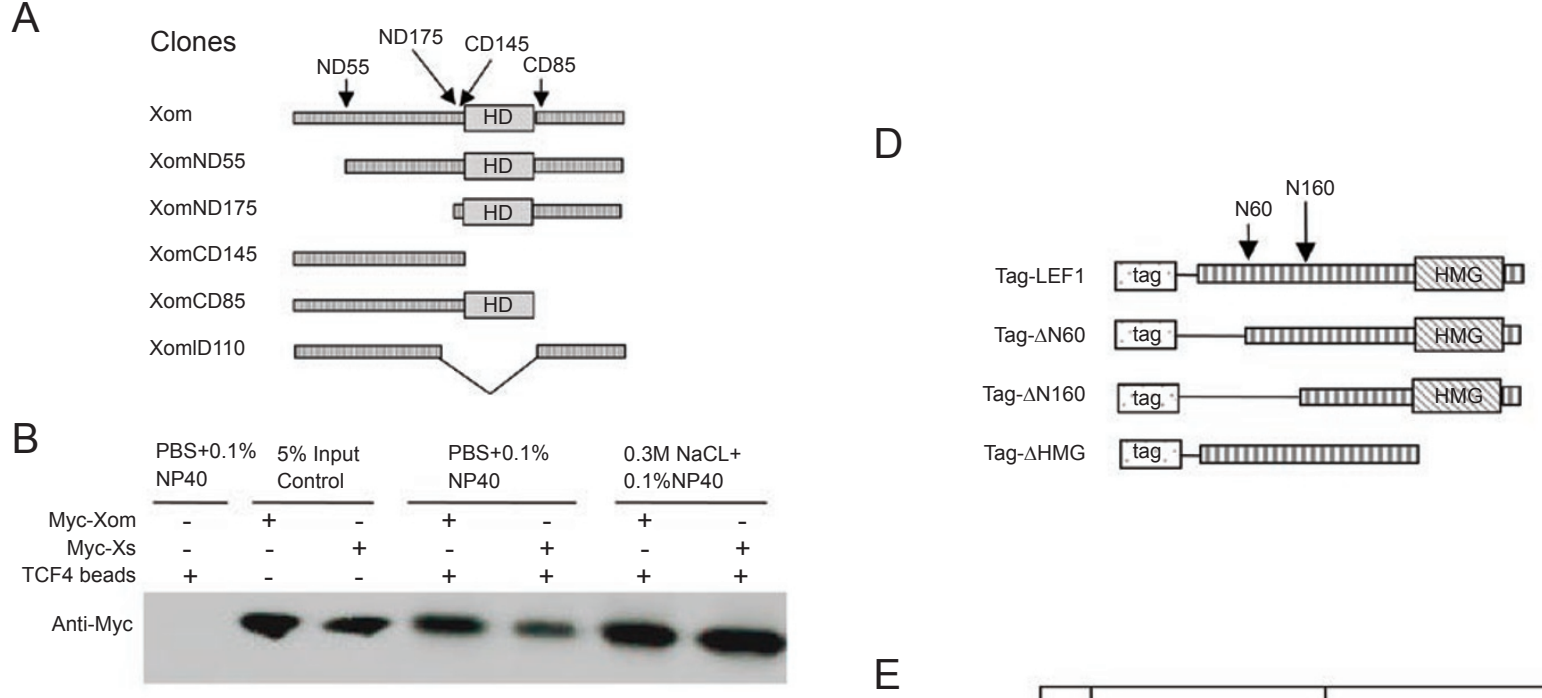

C

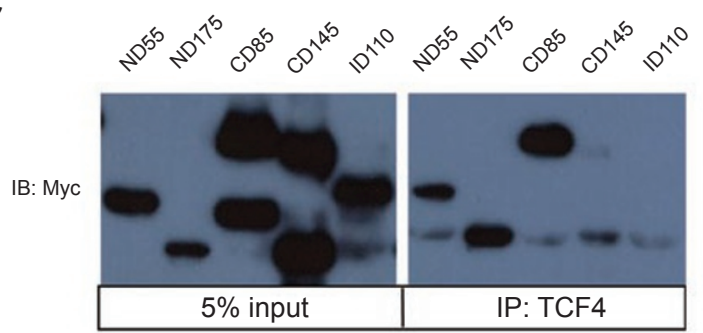

E

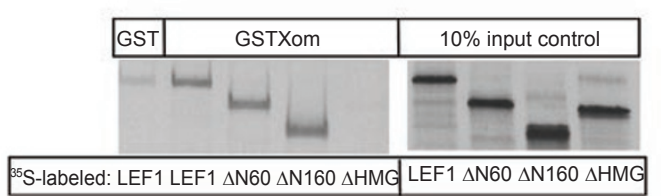

Figure 2 Interaction between Xom and LEF1/TCF factors. (A) Xom and its deletion mutants used in the study of Xom and LEF1/ TCF interaction. (B, C) Interaction between Xom and TCF4 in cells. HCT116 cells were transfected with plasmids encoding myctagged Xom, stable Xom (myc-Xs) or Xom deletion mutants. The endogenous TCF4 was immunoprecipitated with anti-TCF4 beads followed by washing with indicated buffers; the precipitates were subjected to subsequent western blot analysis using the anti-myc antibody. (D) Delineation of LEF1 and LEF1 deletion mutants. (E) In vitro pull-down experiments of GST or GST-Xom with ${ }^{35} \mathrm{~S}$ labeled LEF1 or its deletion mutants; $10 \%$ of loading input is indicated. 
A

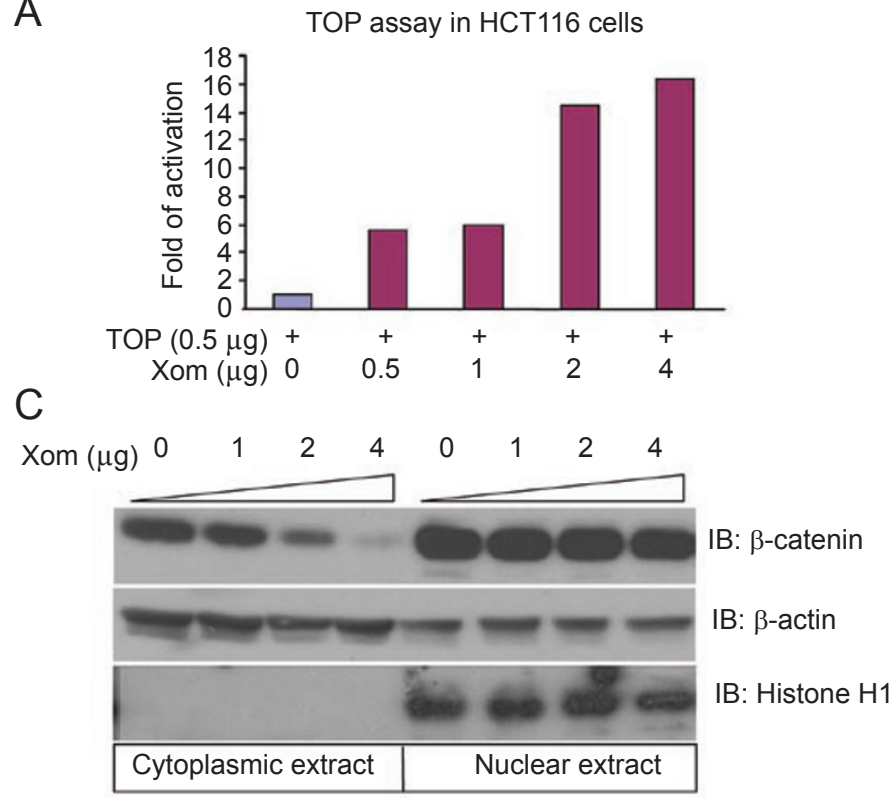

B

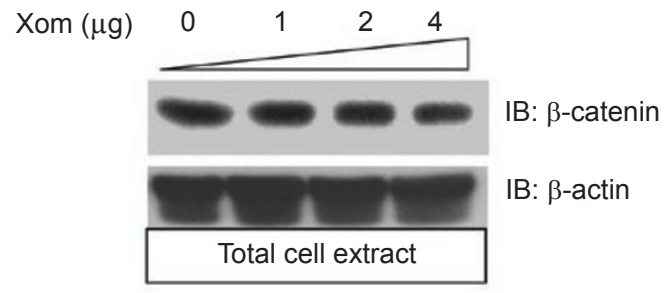

D

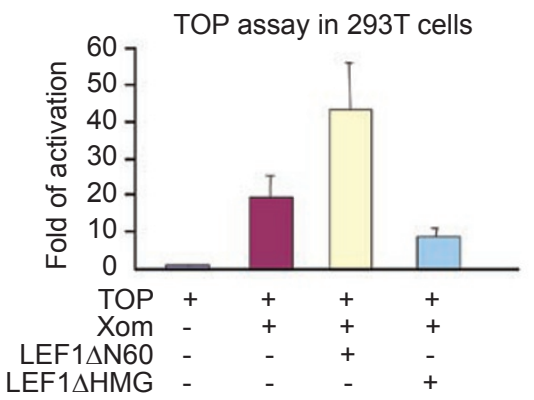

Figure 3 Activation of LEF1/TCF-mediated transcription by Xom is not due to $\beta$-catenin accumulation. (A) Xom expression activates LEF1/TCF reporter in HCT116 cells in a concentration-dependent manner. (B) Effect of Xom expression on total $\beta$-catenin protein levels in HCT116 cells. $2 \times 10^{6}$ HCT 116 cells were transfected with a plasmid encoding Xom. Total cell extracts were harvested with RIPA buffer $48 \mathrm{~h}$ after transfection. The level of $\beta$-catenin in each sample was determined by western blot analysis. $\beta$-Actin was used as the loading control. (C) Effect of Xom expression on intracellular $\beta$-catenin level and distribution. Proteins in HCT116 cells transfected with Xom were fractionated into cytoplasmic and nuclear portions. The $\beta$-catenin level in each portion was determined. $\beta$-Actin was used as the loading control; Histone H1 was used as an indicator of the efficiency of fractionation. (D) Effects of LEF1 deletion mutants on Xom transactivation of TOPflash in 293T cells. Luciferase activities in cells expressing TOPflash alone were defined as one unit. Error bars indicate standard deviations of representative experiments carried out in triplicate.

cells (and later 293T cells) (Figure 1B), Xom expression induced the transcription of the TOPflash constructs in these non-embryonic mammalian cells, indicating that Xom exerts a general induction effect on LEF1/TCF-mediated transcription. Again, the specificity of Xom transactivation of LEF1/TCF-mediated transcription was indicated by the finding that Xom failed to activate the FOPflash reporter construct in these tissue culture experiments (data not shown).

\section{Xom binds to LEF1/TCF factors in vivo and in vitro}

Binding of LEF1/TCFs to the promoter of TOPflash is required for the activation of the reporter construct; thus, the finding that expression of Xom activates TOPflash but not FOPflash raised the possibility that Xom activates the TOPflash reporter through interaction with LEF/TCFs. We tested this hypothesis by determining whether Xom physically interacts with LEF1/TCFs. We carried out co-immunoprecipitation experiments in HCT116 cells transiently transfected with myc-Xom. We found that immunoprecipitation of endogenous TCF4 with anti-TCF4 antibodies co-precipitated the Xom protein (Figure 2A and 2B). The in vivo association between Xom and TCF4 appears to be strong and could not be dissociated with $300 \mathrm{mM} \mathrm{NaCl}$ and $0.1 \%$ NP40. Our previous investigations showed that Xom is an unstable protein and that Xom Ser140/144 to alanine mutation renders the Xom mutant (Xs) resistant to ubiquitin-mediated proteolysis [26]. To determine whether Ser140/144 to alanine mutation interferes with the interaction between Xom and LEF1/TCF-factors, we have also included myc-tagged Xs in the co-immunoprecipitation experiments. Our results showed that myc-Xs binds to TCF4 as efficiently as the wild-type Xom (Figure 2B), indicating that the Ser140/144 to alanine mutation does not interfere with the interaction between Xom and LEF1/TCF-factors. To determine the domain of Xom that mediates its interaction with LEF1/TCFs, we made serial deletion mutants of Xom and tested their interactions with TCF4 in vivo (Figure 2A and 2C). All Xom mutants that carry the homeobox region co-immuno-precipitated with anti-TCF4 beads, while those that lack the homeodomain did not. These data indicate that the homeodomain of 
Xom plays a critical role in mediating complex formation between Xom and LEF1/TCF factors. Given that the homeobox domain is the DNA-binding domain of Xom, to rule out the possibility that the observed interaction between Xom and TCF4 is mediated indirectly by nucleic acids, we included ethidium bromide (EtB, at $20 \mathrm{mg} / \mathrm{ml}$ ) in the pull-down assays [29]. We found that EtB did not interrupt the interaction between TCF4 and Xom or Xom mutants that carry the homeodomain, indicating that the interaction between Xom and TCF4 is not dependent on their potential association with DNA (data not shown). Following the demonstration of complex formation between Xom and TCF4 in vivo, we went further to identify the critical domains of LEF1/TCFs involved in the interaction with Xom. At least four members of the LEF1/TCF family have been identified in vertebrates (LEF1, TCF1, TCF3 and TCF4), and LEF1 shows an expression pattern similar to that of Xom. Zygotic transcription of LEF1 starts after the onset of the MBT and is enriched at the ventral-caudal side of the animal [20]. Genetic data indicate that LEF1 is essential for ventral-posterior patterning [23], consistent with the notion that LEF1 might be a potential candidate as the Xom-interacting protein. We therefore tested whether Xom interacts with LEF1 and explored the critical domain of LEF1 involved in the interaction through deletion analysis (Figure 2D and 2E). During our pilot cotransfection studies, we found that very few cells co-expressed Xom and full-length LEF1 (data not shown); therefore, we probed the potential interaction between Xom and LEF1 through an in vitro binding assay. When in vitro translation products of ${ }^{35} \mathrm{~S}$-labeled LEF1 or its deletion mutants were mixed with GST-Xom or GST alone, LEF1 was pulled down by GST-Xom but not by the control GST (Figure 2E). The C-terminal-deleted mutant LEF1 $\triangle \mathrm{HMG}$ appeared to have much less affinity for Xom, whereas the $\mathrm{N}$-terminal-deleted mutants, LEF $1 \triangle \mathrm{N} 60$ and LEF $1 \triangle \mathrm{N} 160$, bound to GST-Xom as effectively as did the wild-type LEF1 (Figure 2E). Thus, the interaction between Xom and LEF1 appears to be dependent on the LEF1 C-terminal motif, a supposition further supported by a LEF1/TCF transactivation assay (Figure 3D). To determine the potential physiological relevance of Xom transactivation of LEF1/TCF-mediated transcription, we examined the effect of Xom on the expression of BMP4 downstream genes, using promoter-luciferase analysis. The Msx2 gene is downstream of BMP4, and its promoter contains both an LEF1/TCF binding site and a BMP4-responsive element (SBE) [30]. Consistent with the potential direct involvement of LEF1/TCFs in BMP/Xom signaling, we found that Xom activates both the wild-type Msx 2 promoter and the SBE-mutated Msx 2 promoter that retains only the functional LEF1/TCF binding site (data not shown).
Xom transactivation of LEF1/TCF-mediated transcription is not mediated through $\beta$-catenin accumulation

Previous studies have shown that LEF1/TCF-mediated transcription is activated by $\beta$-catenin [31-33]. To explore the mechanism of Xom activation of LEF1/TCF-mediated transcription and to rule out the possibility that Xom transactivation of LEF1/TCF-mediated transcription is through an increase in $\beta$-catenin protein level or nuclear translocation, we examined the effect of Xom expression on the protein level and intracellular distribution of $\beta$-catenin in HCT116 cells. As shown in Figure 3A-C, whereas Xom expression activates LEF1/TCF-mediated transcription in a concentration-dependent manner (Figure 3A), there is no corresponding increase (rather, a small decrease was observed) in the level of total intracellular $\beta$-catenin protein (Figure 3B). In addition, when the total intracellular proteins were fractionated into the cytoplasmic and nuclear portions, no significant increase of nuclear $\beta$-catenin was observed upon Xom expression (rather, a small decrease was observed) (Figure 3C). As shown in Figure 2, the domain of LEF1 that interacts with Xom locates at the C-terminal region of LEF1, which is separated from the $\mathrm{N}$-terminal domain involved in interaction with $\beta$-catenin. Consistent with the notion that Xom transactivates LEF1/ TCF-mediated transcription through their direct interaction, we found that LEF $1 \triangle \mathrm{N} 60$, a dominant-negative LEF1 mutant that is known to block $\beta$-catenin transactivation of LEF1/TCF-mediated transcription but retains the ability to interact with Xom, promotes Xom transactivation of the LEF1/TCF-responsive reporter in the TOPflash assay (Figure 3D). In comparison, the LEF1 $\triangle$ HMG mutant, which fails to interact with Xom, reduces Xom transactivation of the LEF1/TCF-responsive reporter (Figure 3D).

\section{Xom transactivates LEF1/TCFs through its $N$-terminal TAD}

The interaction between Xom and LEF1 allowed us to further explore the molecular mechanism underlying Xom transactivation of LEF1/TCF-mediated transcription. Previous studies have shown that $\beta$-catenin binds to TCFs through its central Armadillo repeats and activates TCFs through its C-terminal motif [25, 34]. Therefore, after identifying the critical domain of Xom involved in LEF1/TCF interaction, we further analyzed the functional domain of Xom involved in activating LEF1/TCF-mediated transcription. When cDNA encoding Xom or its deletion mutants was co-transfected with the reporter construct of TOPflash into 293T cells, expression of both Xom and its Cterminal deletion mutant XomCD85 activated the TOPflash reporter by almost 10 -fold comparing with control cells transfected with TOPflash only (Figure 5A). In contrast, the ability to activate the LEF1/TCF-responsive reporter 
A

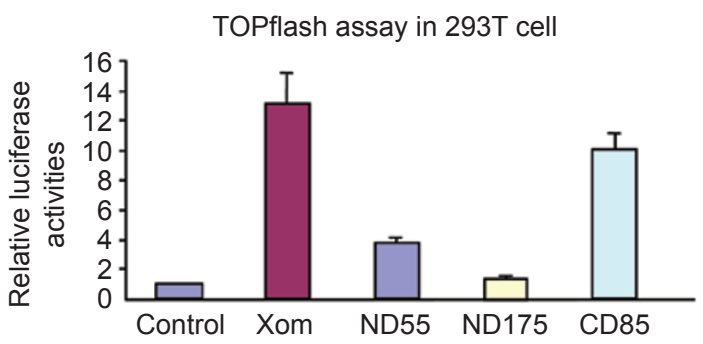

C

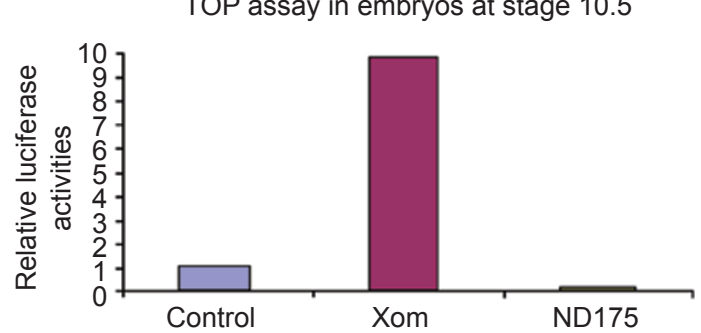

$E$

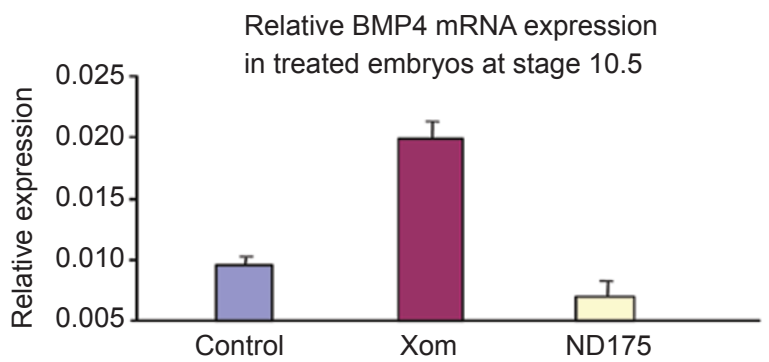

$\mathrm{B}$

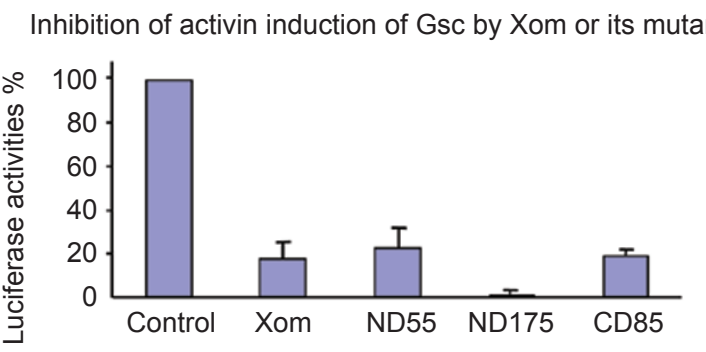

D

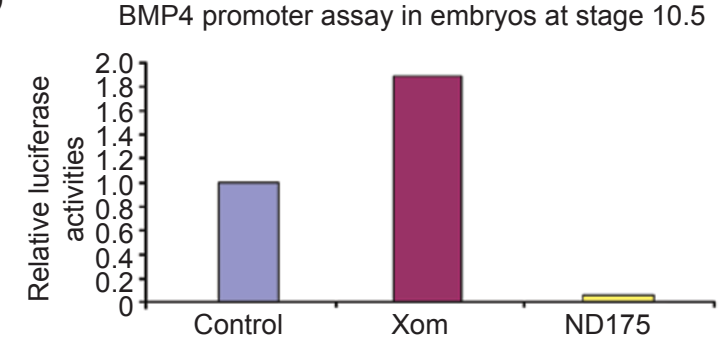

$\mathrm{F}$

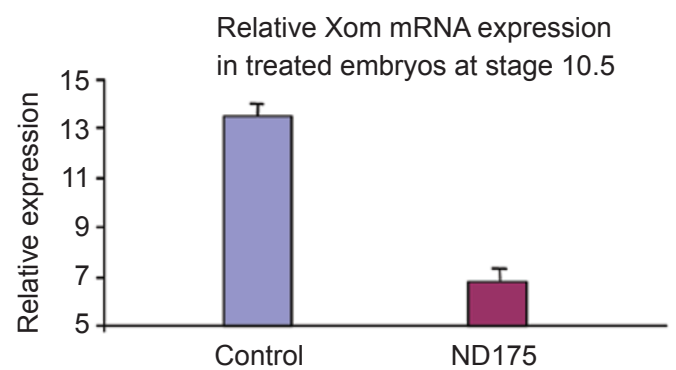

Figure 4 Identification of Xom N-terminal TAD. (A) 293T cells were cotransfected with TOPflash reporter construct and plasmids encoding Xom or its deletion mutants. The effects of Xom or its mutants on LEF1/TCF-mediated transcription were determined after $48 \mathrm{~h}$, using the TOPflash assay. Luciferase activity in cells expressing TOPflash alone was defined as one unit. Error bars indicate standard deviations of representative experiments carried out in triplicate. (B) Effect of Xom or its deletion mutants on Activin-induced transactivation of Goosecoid promoter. One blastomere of embryos at the two-cell stage was injected with the Goosecoid-luciferase reporter construct (100 pg) and mRNA encoding Activin (50 pg), either alone or in combination with mRNA encoding Xom or its deletion mutants. The embryos were cultured to stage 10.5 , and the level of luciferase activity was measured as described. The luciferase activity in embryos injected with Goosecoid-luciferase reporter construct and Activin alone was defined as 100. (C, D) Effects of Xom or XomND175 on transcription activation of the TOPflash and BMP4 promoter -luciferase reporters. Each blastomere of embryos at the two-cell stage was injected with $0.1 \mathrm{ng}$ of indicated reporter construct in the presence or absence of $0.4 \mathrm{ng}$ of mRNA encoding Xom or XomND175. The embryos were cultured to stage 10.5, and luciferase assays were performed as described. Representative data of duplicated experiments were presented. (E, F) Effects of Xom or XomND175 on the expression of endogenous BMP4 and Xom. Each blastomere of embryos at the two-cell stage was injected with $0.4 \mathrm{ng}$ of mRNA encoding Xom or XomND175 as indicated. The embryos were cultured to stage 10.5, and total RNA was extracted. The levels of BMP4 (E) and Xom (F) mRNAs in these embryos were determined by Q-PCR and normalized to the mRNA level of Histone H4.

was significantly diminished in the N-terminal deletion mutants of Xom, XomND55 and XomND175. XomND175 in particular retains little if any ability to activate the LEF1/ TCF-responsive reporter (Figure 4A). Consistent with the model whereby Xom transactivates LEF1/TCF-mediated transcription through direct interaction, we found that the Xom mutant lacking the LEF1/TCF-interaction motif failed to activate LEF1/TCF-mediated transcription during early embryogenesis (data not shown). Xom is a known transcriptional repressor of dorsal gene expression. To determine whether the dual transcriptional function of Xom is independent of each other, we further examined the effect of these Xom deletion mutants on dorsal gene expression during early embryogenesis. For the in vivo experiments, 
A
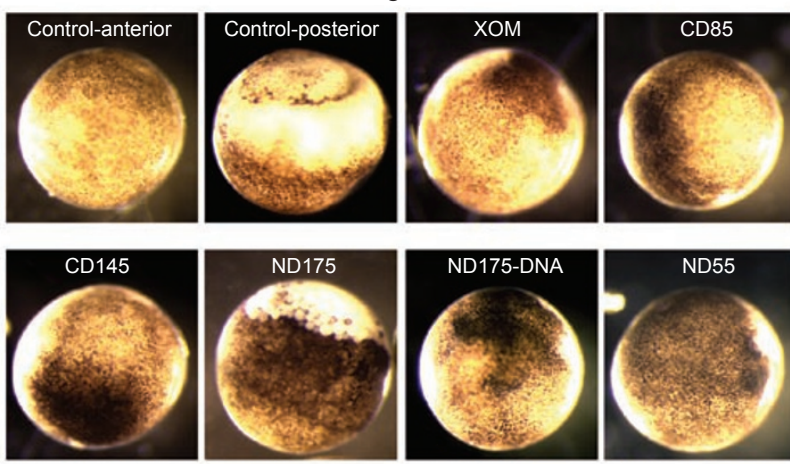

C
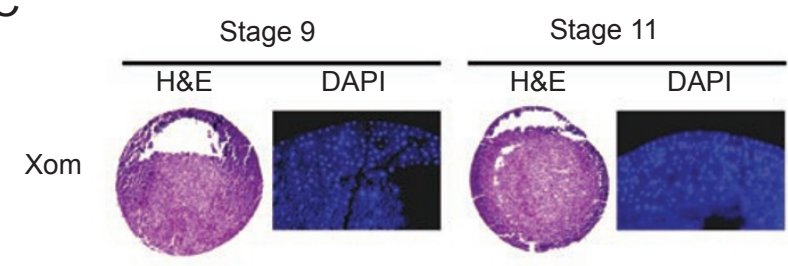

ND175
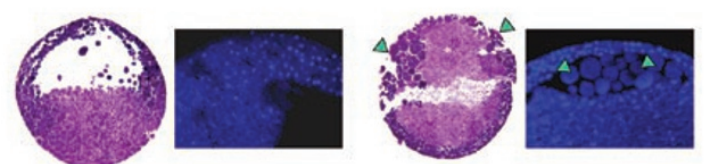

B

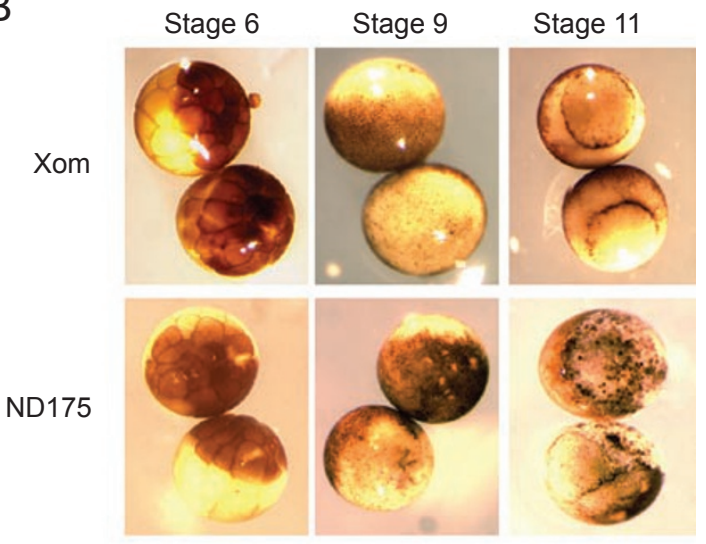

D

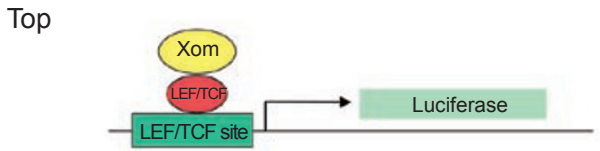

Bottom

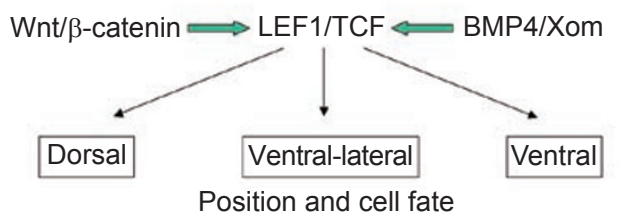

Figure 5 Deletion of Xom TAD has catastrophic effects during gastrulation. (A) Phenotype of gastrula-stage embryos injected with the indicated mRNA $(0.4 \mathrm{ng})$ or cDNA $(0.1 \mathrm{ng})$ in one of the two ventral blastomeres at the four-cell stage. (B) Stage-specific effect of XomND175. One of the two ventral blastomeres at the four-cell stage was injected with mRNA (0.4 ng) encoding XomND175, and the effects were followed. (C) H\&E and DAPI staining of thin sections of embryos injected with mRNA encoding Xom or XomND175 as detailed above. Arrows indicate area of loss of cellular structures. (D) Schematic models for Xom transactivation of LEF1/TCF-mediated transcription (top) and for the role of LEF1/TCF as the convergence point integrating the signaling by Wnt/ $\beta$ catenin and BMP4/Xom pathways in cell fate determination during early embryogenesis (bottom).

mRNA encoding Xom or its deletion mutants were mixed with mRNA encoding Activin, and then co-injected with the Gsc-promoter-luciferase construct into the embryos at the two-cell stage. We found that both the N-terminal deletion and the C-terminal deletion mutants of Xom strongly inhibited Activin-induced activation of the Gsc promoter (Figure 4B). These data indicate that the function of Xom TAD is independent of the Xom repressor function, and that the Xom TAD resides in its N-terminal region, a notion that was further supported by TOPflash assays in early embryos (Figure 4C).

\section{Xom N-terminal TAD is requried for ventral signaling}

Xom is emerging as an essential cell fate determination factor during mesoderm differentiation to promote the expression of ventral specific genes [6]. It has been proposed that Xom forms a positive re-enforcement loop with BMP4 to promote ventral cell fate [3]. To determine whether the Xom N-terminal TAD plays a role in Xom transactivation of ventral specific genes, we examined the effect of XomND175 on transactivation of the BMP4 promoter during early embryogenesis (the BMP4-luciferase construct was a generous gift of Dr J Feng $[35,36]$ ). We found that, in contrast to Xom, XomND175 failed to transactivate the BMP4 promoter (Figure 4D). Moreover, in comparison with the luciferase activities in control embryos, embryos injected with XomND175 revealed less luciferase activities, suggesting that expression of XomND175 exerts a dominant-negative effect to block the promotion of BMP4 expression by endogenous Xom. To further determine the effect of Xom TAD on the expression of genes downstream of BMP4 signaling, each of 
the two blastomeres of embryos at the two-cell stage was injected with mRNA encoding Xom or XomND175. The embryos were allowed to develop until the gastrula stage (stage 10.5) and total mRNAs were extracted from the injected embryos and non-injected control embryos. The levels of BMP4 and Xom expression were determined by Q-PCR, using Histone-4 as an internal control. Consistent with its role in transactivation of ventral gene expression, expression of wild-type Xom increased the expression of BMP4 (Figure 4E). In contrast, expression of XomND175 failed to enhance the expression of BMP4, suggesting that the Xom TAD is required for its transactivation of BMP4 expression. Moreover, consistent with the disruption of the positive auto feedback loop of BMP4 and Xom, expression of XomND175 exerted a strong inhibitory effect on the expression of Xom itself (Figure 4F). Together with the notion that LEF1/TCF binding sites in the promoters of ventral specific genes are required for their responsiveness to BMP4 signaling and that loss of function of LEF1 leads to ventral defects $[23,24]$, our findings suggest that Xom transactivation of LEF1/TCF-mediated transcription is likely to play a critical role in ventral signaling and ventral cell fate determination.

\section{Xom transactivation of LEF1/TCFs is essential for gas- trulation}

Our biochemical and mutagenesis studies revealed that Xom possesses a LEF1/TCF-TAD in its N-terminal region. We have identified a Xom mutant, XomND175, which possesses negligible ability to transactivate LEF1/TCF but retains the transcriptional repressor function. To determine the potential physiological function of Xom transactivation of LEF1/TCF-mediated transcription during early embryogenesis, we used the loss-of-function approach and explored the effect of XomND175 expression on embryogenesis. We obtained mRNAs encoding Xom, XomND175, and other Xom deletion mutants through in vitro transcription. The mRNAs were diluted to desired concentration with distilled water and injected into one of the two ventral blastomeres at the four-cell stage as indicated (Figure $5 \mathrm{~A}$ ). We observed that embryos injected with mRNA of XomND175, but not other Xom mutants, showed catastrophic effects during gastrulation (Figure 5A). Moreover, the effects of XomND175 appeared to be stage-specific. Unlike XomND175 mRNA, XomND175 cDNA caused little, if any, abnormalities during gastrulation (Figure 5A). Closer inspection showed that the embryos injected with XomND175 mRNA progressed through the cleavage and pregastrulation stages; however, as gastrulation begins, many large whitish gray-colored cells appear in the injected side (Figure 5B). As gastrulation proceeds, these cells gradually lose a defined cellular appearance, giving the embryos a "rotten" appearance. To define the cellular and histological changes associated with XomND175 expression, we fixed the XomND175-mRNA-injected and control embryos, sliced them into $10-\mu \mathrm{M}$ thin sections, and stained them with H\&E and DAPI. Little histological change was associated with ND175 expression at the cleavage and pregastrulation stages, but many large rounded-up structures were seen at the gastrulation stage, which appeared to be dead cells. None of these large rounded-up structures in ND175-injected embryos stained with DAPI, suggesting that they had lost normal cellular structures, such as the nucleus (Figure 5C, arrow head). The embryos appeared to be very sensitive to the effect of XomND175. The 50\% effective penetration rate was achieved with less than 50 pg of XomND175 mRNA (data not shown). The finding that expression of a dominant-negative Xom mutant that lacks TAD led to embryonic arrest at the gastrulation stage suggests that Xom transactivation of LEF1/TCF-mediated transcription is essential for gastrulation. Similar findings have been noted for other transcriptional factors related to BMP4 signaling, such as Bix3 [37].

\section{Discussion}

LEF1/TCFs are essential cell fate determination factors whose transcriptional activities are directed by associated factors. Previous studies have described the role of $\beta$-catenin as the transcriptional activator of LEF1/TCFs and the essential functions of $\beta$-catenin activated LEF1/ TCF-mediated transcription in dorsoventral patterning, ontogenesis and malignant transformation. Our current investigation indicates that Xom, a homeobox transcription factor with dual roles of transcriptional repressor and activator functions, activates LEF1/TCF-mediated transcription. We found that Xom forms a complex with LEF1/TCF factors and activates LEF1/TCF-mediated transcription through its N-terminal TAD. Our finding that a dominant-negative Xom mutant that lacks the TAD leads to embryonic development arrest at the gastrulation stage suggests that Xom transactivation of LEF1/TCF-mediated transcription is essential for cell fate determination during early embryogenesis.

LEF1/TCFs are architectural transcription factors that may induce sharp bending in the DNA helix $[31,38,39]$. Multiple transcriptional factors were found to modulate the $\beta$-catenin/LEF1/TCF signaling [40-42]. The findings that Xom, a transcription factor that offsets the $\beta$-catenin effects on cell fate, binds to LEF1/TCF factors and transactivates LEF1/TCF-mediated transcription through its N-terminal $\mathrm{TAD}$, therefore, presents a new paradigm to understand LEF1/TCF-mediated transactivation and its consequence in cell fate determination. Distinguished from $\beta$-catenin, 
Xom functions also as a transcriptional repressor. Thus, the finding that Xom transactivates LEF1/TCF-mediated transcription reveals a potential mechanism to account for the dual transcriptional functions of Xom. We have mapped the Xom TAD to the N-terminal region of Xom. We have yet to determine whether Xom TAD interacts with the basic transcriptional machinery directly or acts through other intermediates. Previous investigations have shown that Smads binds to both LEF1/TCFs and Xom/Vent-2 [30, 4244]. Thus, possibilities exist that Smads may modulate Xom transactivation of LEF1/TCF target genes, similar to their involvement in $\beta$-catenin transactivation of LEF1/TCFmediated transcription $[30,42,44]$. Further investigations would help to decipher how multiple signaling pathways are integrated in cell fate determination.

Xom and BMP4 have been postulated to form a positive re-enforcement loop to promote ventral cell fate and to inhibit dorsal cell fate. Previous studies have shown that Xom and BMP4 promote the expression of each other and enhance the expression of ventral genes, such as the BMP4, Wnt8, Vent1 and Xom itself [2, 3]. The molecular mechanism of Xom transactivation of ventral genes was not clear. Recent mutagenesis analysis of ventral gene promoters revealed the critical role of the LEF1/TCF binding sites of these promoters in their responsiveness to BMP4 signaling [24]. Thus, our findings that Xom binds to LEF1/TCF factors and transactivates LEF1/TCF-mediated transcription suggest a mechanistic explanation for Xom-mediated activation of ventral gene expression. The potential involvement of Xom transactivation of LEF1/TCF-mediated transcription in vivo is consistent with the findings of Ramel and Lekven, [45] who showed that depletion of Xom-like molecules (such as Vent and Vox) by morpholino reduces TOP activities in some embryos. The essential role of Xom transactivation of LEF1/TCF-mediated transcription during early embryogenesis was implicated by the catastrophic effect of expressing the dominant-negative Xom that lacks the TAD (XomND175). XomND175 therefore may serve as a useful probe to explore the essential genes and signaling pathways underlying Xom induced cell fate determination during early embryogenesis.

The molecular mechanism underlying the establishment of dorsoventral asymmetry from the symmetrical fertilized eggs constitutes a fundamental question that is of broad interest in both the biology of early development as well as pathogenesis of a variety of diseases, such as neogenesis and degeneration. The results of previous investigations revealed the critical role of dorsal and ventral organizers in the establishment of dorsoventral asymmetry. Previous investigations have defined the role of LEF1/TCFs as a component of $\mathrm{Wnt} / \beta$-catenin signaling pathway during the establishment of dorsal organizing center. Our current investigations present several lines of evidence indicating that LEF1/TCFs also participate in the BMP4/Xom signaling of the ventral signal center (Figure 5D, upper panel). The LEF1/TCFs, a prior known component of the Wnt/ $\beta$ catenin pathway, therefore, may function as the point of convergence to integrate the combined signaling effects of the Wnt/ $\beta$-catenin and BMP4/Xom pathways during the establishment of the dorsal-ventral axis (Figure 5D, lower panel). Both $\beta$-catenin and Xom are activators of LEF1/ TCF-mediated transcription; therefore, $\beta$-catenin and Xom could potentially function in a synergistic or antagonizing fashion in a context-dependent manner. The complexity of the promoters of BMP and Wnt downstream genes and the availability of other participating co-factors may play a critical role in the readout of the combined signaling effects of these two pathways. It has been found that the Wnt and BMP pathways synergize to promote the expression of ventral posterior genes, such as $T b x 6$, but counteract to regulate the expression of dorsal genes, such as geminin $[46,47]$. Extensive investigations have identified multiple extracellular molecules as the mediator of the cross-talk between the dorsal and ventral signaling; our identification of the LEF1/TCF factors as the convergence point of the dorsal and ventral signaling pathway, therefore, presents a new paradigm in understanding the intracellular integration of the dorsal and ventral signaling in cell fate determination during early embryogenesis.

\section{Acknowledgments}

We thank M Kirschner for critical discussions; B Vogelstein, J Feng and C Sirard for providing critical reagents; M Whitman, B Olsen, M Jeans, K Gideon, S Colgan and R Blumberg for the use of their facilities; and Y Shi for critical reading of the manuscript. Z Zhu was supported by grants from the National Institutes of Health and start-up funds from Brigham and Women's Hospital.

\section{References}

1 Ladher R, Mohun TJ, Smith JC, Snape AM. Xom: a Xenopus homeobox gene that mediates the early effects of BMP-4. Development 1996; 122:2385-2394.

2 Onichtchouk D, Gawantka V, Dosch R, et al. The Xvent-2 homeobox gene is part of the BMP-4 signalling pathway controlling dorsoventral patterning of Xenopus mesoderm. Development 1996; 122:3045-3053.

3 Schmidt JE, von Dassow G, Kimelman D. Regulation of dorsalventral patterning: the ventralizing effects of the novel Xenopus homeobox gene Vox. Development 1996; 122:1711-1721.

4 Papalopulu N, Kintner C. A Xenopus gene, Xbr-1, defines a novel class of homeobox genes and is expressed in the dorsal ciliary margin of the eye. Dev Biol 1996; 174:104-114.

5 Onichtchouk D, Glinka A, Niehrs C. Requirement for Xvent-1 
and Xvent-2 gene function in dorsoventral patterning of Xenopus mesoderm. Development 1998; 125:1447-1456.

6 Koide T, Hayata T, Cho KW. Xenopus as a model system to study transcriptional regulatory networks. Proc Natl Acad Sci USA 2005; 102:4943-4948.

7 Melby AE, Clements WK, Kimelman D. Regulation of dorsal gene expression in Xenopus by the ventralizing homeodomain gene Vox. Dev Biol 1999; 211:293-305.

8 Trindade M, Tada M, Smith JC. DNA-binding specificity and embryological function of Xom (Xvent-2). Dev Biol 1999; 216:442-456.

9 Hurlstone A, Clevers H. T-cell factors: turn-ons and turn-offs. EMBO J 2002; 21:2303-2311.

10 Roose J, Molenaar M, Peterson J, et al. The Xenopus Wnt effector XTcf-3 interacts with Groucho-related transcriptional repressors. Nature 1998; 395:608-612.

11 Brantjes H, Roose J, van De Wetering M, Clevers H. All Tcf HMG box transcription factors interact with Groucho-related co-repressors. Nucleic Acids Res 2001; 29:1410-1419.

12 Cavallo RA, Cox RT, Moline MM, et al. Drosophila Tef and Groucho interact to repress Wingless signalling activity. Nature 1998; 395:604-608.

13 Waltzer L, Bienz M. Drosophila CBP represses the transcription factor TCF to antagonize wingless signalling. Nature 1998; 395:521-525.

14 Brannon M, Brown JD, Bates R, Kimelman D, Moon RT. XCtBP is a XTcf-3 co-repressor with roles throughout Xenopus development. Development 1999; 126:3159-3170.

15 Harland R, Gerhart J. Formation and function of Spemann's organizer. Annu Rev Cell Dev Biol 1997; 13:611-667.

16 Brannon M, Gomperts M, Sumoy L, Moon RT, Kimelman D. A beta-catenin/XTcf-3 complex binds to the siamois promoter to regulate dorsal axis specification in Xenopus. Genes Dev 1997; 11:2359-2370.

17 Laurent MN, Blitz IL, Hashimoto C, Rothbacher U, Cho KW. The Xenopus homeobox gene twin mediates Wnt induction of goosecoid in establishment of Spemann's organizer. Development 1997; 124:4905-4916.

18 McKendry R, Hsu SC, Harland RM, Grosschedl R. LEF-1/TCF proteins mediate wnt-inducible transcription from the Xenopus nodal-related 3 promoter. Dev Biol 1997; 192:420-431.

19 Barker N, Morin PJ, Clevers H. The Yin-Yang of TCF/betacatenin signaling. Adv Cancer Res 2000; 77:1-24.

20 Molenaar M, Roose J, Peterson J, Venanzi S, Clevers H, Destree O. Differential expression of the HMG box transcription factors XTcf-3 and XLef-1 during early Xenopus development. Mech Dev 1998; 75:151-154.

21 Oosterwegel M, van de Wetering M, Timmerman J, et al. Differential expression of the HMG box factors TCF-1 and LEF-1 during murine embryogenesis. Development 1993; 118:439448.

22 Galceran J, Farinas I, Depew MJ, Clevers H, Grosschedl R. Wnt3a-/--like phenotype and limb deficiency in Lef1(-/-)Tcf1(-/-) mice. Genes Dev 1999; 13:709-717.

23 Roel G, Hamilton FS, Gent Y, Bain AA, Destree O, Hoppler S. Lef-1 and Tcf-3 transcription factors mediate tissue-specific Wnt signaling during Xenopus development. Curr Biol 2002; 12:1941-1945.

24 Karaulanov E, Knochel W, Niehrs C. Transcriptional regulation of BMP4 synexpression in transgenic Xenopus. EMBO J 2004; 23:844-856.

25 van de Wetering M, Cavallo R, Dooijes D, et al. Armadillo coactivates transcription driven by the product of the Drosophila segment polarity gene dTCF. Cell 1997; 88:789-799.

26 Zhu Z, Kirschner M. Regulated proteolysis of Xom mediates dorsoventral pattern formation during early Xenopus development. Dev Cell 2002; 3:557-568.

27 Kiecker C, Niehrs C. A morphogen gradient of Wnt/beta-catenin signalling regulates anteroposterior neural patterning in Xenopus. Development 2001; 128:4189-4201.

28 Korinek V, Barker N, Morin PJ, et al. Constitutive transcriptional activation by a beta-catenin-Tcf complex in APC-/- colon carcinoma. Science 1997; 275:1784-1787.

29 Lai JS, Herr W. Ethidium bromide provides a simple tool for identifying genuine DNA-independent protein associations. Proc Natl Acad Sci USA 1992; 89:6958-6962.

30 Hussein SM, Duff EK, Sirard C. Smad4 and beta-catenin coactivators functionally interact with lymphoid-enhancing factor to regulate graded expression of Msx2. J Biol Chem 2003; 278:48805-48814.

31 Behrens J, von Kries JP, Kuhl M, et al. Functional interaction of beta-catenin with the transcription factor LEF-1. Nature 1996; 382:638-642.

32 Huber O, Korn R, McLaughlin J, Ohsugi M, Herrmann BG, Kemler R. Nuclear localization of beta-catenin by interaction with transcription factor LEF-1. Mech Dev 1996; 59:3-10.

33 Molenaar M, van de Wetering M, Oosterwegel M, et al. XTcf-3 transcription factor mediates beta-catenin-induced axis formation in Xenopus embryos. Cell 1996; 86:391-399.

34 Orsulic S, Peifer M. An in vivo structure-function study of Armadillo, the beta-catenin homologue, reveals both separate and overlapping regions of the protein required for cell adhesion and for wingless signaling. J Cell Biol 1996; 134:1283-1300.

35 Zhang J, Tan X, Contag CH, et al. Dissection of promoter control modules that direct Bmp4 expression in the epithelium-derived components of hair follicles. Biochem Biophys Res Commun 2002; 293:1412-1419.

36 Ogawa T, Kapadia H, Feng JQ, Raghow R, Peters H, D’Souza $\mathrm{RN}$. Functional consequences of interactions between Pax 9 and Msx1 genes in normal and abnormal tooth development. J Biol Chem 2006; 281:18363-18369.

37 Trindade M, Messenger N, Papin C, et al. Regulation of apoptosis in the Xenopus embryo by Bix3. Development 2003; 130:46114622.

38 van Noort M, Clevers H. TCF transcription factors, mediators of Wnt-signaling in development and cancer. Dev Biol 2002; 244:1-8.

39 Radtke F, Clevers H. Self-renewal and cancer of the gut: two sides of a coin. Science 2005; 307:1904-1909.

40 Takemaru KI, Moon RT. The transcriptional coactivator CBP interacts with beta-catenin to activate gene expression. J Cell Biol 2000; 149:249-254.

41 Kramps T, Peter O, Brunner E, et al. Wnt/wingless signaling requires BCL9/legless-mediated recruitment of pygopus to the nuclear beta-catenin-TCF complex. Cell 2002; 109:47-60.

42 Nishita M, Hashimoto MK, Ogata S, et al. Interaction between Wnt and TGF-beta signalling pathways during formation of Spemann's organizer. Nature 2000; 403:781-785. 
43 Henningfeld KA, Friedle H, Rastegar S, Knochel W. Autoregulation of Xvent-2B; direct interaction and functional cooperation of Xvent-2 and Smad1. J Biol Chem 2002; 277:2097-2103.

44 Labbe E, Letamendia A, Attisano L. Association of Smads with lymphoid enhancer binding factor $1 / \mathrm{T}$ cell-specific factor mediates cooperative signaling by the transforming growth factor-beta and wnt pathways. Proc Natl Acad Sci USA 2000; 97:8358-8363.

45 Ramel MC, Lekven AC. Repression of the vertebrate organizer by Wnt 8 is mediated by Vent and Vox. Development 2004; 131:3991-4000.

46 Szeto DP, Kimelman D. Combinatorial gene regulation by Bmp and Wnt in zebrafish posterior mesoderm formation. Development 2004; 131:3751-3760.

47 Taylor JJ, Wang T, Kroll KL. Tcf- and Vent-binding sites regulate neural-specific geminin expression in the gastrula embryo. Dev Biol 2006; 289:494-506. 